\title{
Narrative review-cardiovascular evaluation before radiotherapy for patients with breast cancer and other malignancies
}

\author{
Wei-Ting Chang ${ }^{1,2,3}$, Yin-Hsun Feng ${ }^{4}$, Zhih-Cherng Chen ${ }^{1}$, Yen-Wen $\mathrm{Wu}^{5,6,7}$ \\ ${ }^{1}$ Division of Cardiology, Department of Internal Medicine, Chi-Mei Medical Center, Tainan, Taiwan; ${ }^{2}$ Department of Biotechnology, Southern \\ Taiwan University of Science and Technology, Tainan, Taiwan; ${ }^{3}$ Institute of Clinical Medicine, College of Medicine, National Cheng Kung \\ University, Tainan, Taiwan; ${ }^{4}$ Division of Oncology, Department of Internal Medicine, Chi-Mei Medical Center, Tainan, Taiwan; ${ }^{5}$ Division of \\ Cardiology, Cardiovascular Medical Center, Far Eastern Memorial Hospital, New Taipei City, Taiwan; ${ }^{6}$ Department of Nuclear Medicine, Far \\ Eastern Memorial Hospital, New Taipei City, Taiwan; ${ }^{7}$ School of Medicine, National Yang Ming Chiao Tung University, Taipei, Taiwan \\ Contributions: (I) Conception and design: WT Chang, YW Wu; (II) Administrative support: All authors; (III) Provision of study materials or patients: \\ All authors; (IV) Collection and assembly of data: WT Chang, YW Wu; (V) Data analysis and interpretation: WT Chang; (VI) Manuscript writing: \\ All authors; (VII) Final approval of manuscript: All authors. \\ Correspondence to: Yen-Wen Wu, MD, PhD. Division of Cardiology, Cardiovascular Medical Center, Far Eastern Memorial Hospital, No. 21, Sec. 2, \\ Nanya S. Rd., Banciao Dist., New Taipei City 220, Taiwan. Email: wuyw0502@gmail.com.
}

Objective: To raise awareness of possible cardiovascular risks among cancer survivors who received radiotherapy and to establish appropriate platforms for screening and monitoring the cardiovascular condition of these patients.

Background: With advances in anti-cancer therapies, an increasing number of cancer patients are surviving for longer after the diagnosis and treatment. However, cancer therapy-associated cardiovascular complications can exacerbate mortality and morbidity. To note, radiation-induced cardiovascular diseases (RICVDs) cause deleterious effects on the heart, especially among survivors of breast cancer or malignancies in the chest cavity.

Methods: Through literature searches, we observed a wide range of cardiovascular complications including heart failure, arrhythmia, valvular and ischemic heart diseases which have been reported depending on the cancer type and therapy. Among different types of malignancies, breast cancer, lung cancer, esophageal cancer and lymphoma may require direct radiotherapy to the heart. Despite a slow clinical course, radiation-related myocardial and vascular damage continuously accumulate. Instead of a rescue strategy, a comprehensive cardiovascular evaluation beforehand may be crucial to prevent the occurrence of cardiovascular complications.

Conclusions: In this review article, we highlighted the importance of cardiovascular evaluation before radiotherapy for patients with breast cancer or other malignancy. Nevertheless, large-scale randomized control studies are crucial for establishing a clinical consensus.

Keywords: Radiotherapy; cardiovascular complication; evaluation; breast cancer

Received: 03 August 2021; Accepted: 04 November 2021; Published: 30 December 2021.

doi: $10.21037 /$ tro-21-21

View this article at: https://dx.doi.org/10.21037/tro-21-21

\section{Radiation-induced cardiovascular diseases (RICVDs)}

Among patients receiving thoracic radiotherapy, cardiovascular diseases are the most frequent non-malignant cause of death (1-4). RICVDs cause a range of deleterious effects on the heart (5-7). Survivors of breast cancer, especially left sided, who receive mediastinal irradiation have been associated with a higher risk of cardiovascular mortality (8). Thoracic radiotherapy can lead to the generation of reactive oxygen species which disrupt DNA 
strands, and secondary inflammatory changes contributing to myocardial fibrosis $(5,7)$. In addition, cytoplasmic swelling, thrombosis, and rupture of the walls have been reported in the endothelial cells in coronary arteries (5-7). The damage potentially caused by thoracic radiation includes microvascular ischemia, disruption of the capillary endothelial framework, injury to differentiated myocytes, deposition of collagen, fibrosis and eventually heart failure $(5,6)$. After exposure to radiation, radiotherapy-related coronary atherosclerosis mainly occurs in the left anterior descending and right coronary arteries, and usually 10 to 15 years post treatment (9). In addition to coronary atherosclerosis, radiotherapy has also been associated with the development of pericarditis and pericardial effusion 6 to 12 months post therapies (9). Moreover, valvular calcification and regurgitation are also frequently observed $(6,9,10)$. Occasionally, fibrosis of the conduction system contributes to conduction abnormalities $(5,11)$. We present the following article in accordance with the Narrative Review reporting checklist (available at https://dx.doi. org/10.21037/tro-21-21).

\section{Prevalence of RICVDs}

Post-thoracic radiotherapy, the estimated incidence of RICVDs is $10-30 \%$ by $5-10$ years $(5,12,13)$. Between 1958 and 2001 in Sweden and Denmark, among 2,168 women who underwent radiotherapy at an average dose to the whole heart of 4.9 Gy (range, 0.03-27.72 Gy) for breast cancer, the rates of major coronary events increased linearly with a mean dose to the heart by $7.4 \%$ per Gy with no apparent threshold (4). The increased risk was seen within the first five years and remained elevated for at least 20 years. Although the proportional rates of RICVDs were similar between patients with and without cardiac risk factors at the time of radiotherapy (4), the absolute rate was higher in those with pre-existing CVD risk factors. Most importantly, the precise prevalence of RICVDs is difficult to determine given a long delay between exposure and clinical manifestations. In addition, the use of concomitant cardiotoxic chemotherapy, continuous improvements in radiation techniques and changes in the treated population also make it difficult to determine the precise prevalence. Hodgkin lymphoma is also sensitive to radiotherapy, and radiation exposure frequently covers the heart (14). Despite a favorable long-term survival, especially for pediatric patients, a higher risk of cardiovascular complications including coronary heart disease, heart failure and valvular heart diseases has been reported to persist for at least 25 years after the initial treatment (14). The combination or sequential use of anthracyclines further augments the risks of heart failure and valvular heart diseases associated with thoracic radiotherapy (15). In addition, given the close proximity of the esophagus to the heart, cardiac exposure is unavoidable, resulting in high doses of radiation (16). In terms of lung cancer, although the locations of lung nodules vary, RICVDs in patients with lung cancer are often overlooked. In a randomized phase 3 study, Bradley et al. compared standard-dose versus high-dose conformal radiotherapy in patients with stage IIIA or IIIB non-smallcell lung cancer (17). Notably, the high-dose radiotherapy was not better than the standard-dose ones and might be potentially harmful. It indicated that among patients with cancer at an advanced stage or with poor survival, concerns regarding RICVDs should still be concerned.

\section{Risk factors for RICVDs}

Age at irradiation for breast cancer has been associated with the risk of the subsequent development of RICVDs $(13,18,19)$, and patients younger than 35 years have been reported to have a relative risk of 6.5 compared to the general population $(13,20)$. Other risk factors include hypertension, diabetes, high body mass index, hyperlipidemia and previous anthracycline-based chemotherapy $(1,4,20)$. In a Surveillance, Epidemiology, and End Results (SEER)-based analysis, Hardy et al. found that the risks of RICVDs in patients receiving radiotherapy alone or combined with chemoradiotherapy were 1.5 - and 2.4-fold higher than those not receiving radiotherapy (21). In addition to patient-associated risk factors, the cumulative dose and its fractioning have also been reported to determine the acute and chronic cardiac effects of radiation therapy $(1,4,20,22)$. Dose restriction to 30 Gy with lower daily fraction, different weighting of radiation fields, and blocking the sub-carinal region have been reported to reduce the incidence of pericarditis from $20 \%$ to $2.5 \%$ (23). Thus, a comprehensive evaluation of cardiovascular risk factors followed by aggressive management appears to play pivotal roles in preventing RICVDs.

\section{Cardiovascular imaging before, during and after radiotherapy}

In cancer patients receiving radiotherapy, cardiovascular abnormalities should be identified prior to treatment 
to minimize cardiovascular comorbidities before anticancer therapies and continuing through survivorship $(4,24)$. Patients with abnormal cardiac functions before radiotherapy should be treated carefully while a multidiscipline team including cardiovascular specialists is recommended (24). In clinical practices, the use of cardiac imaging is usually driven by typical symptoms of angina, orthopnea or edema $(12,20)$. However, given that most patients present with atypical symptoms, aggressive screening and follow-up cardiac imaging is crucial for the evaluation and early detection of cardiovascular disorders (1,25). Providing a real-time approach, echocardiography plays a pivotal role in evaluating structural and hemodynamic abnormalities of the heart (26). Using the biplane Simpson's method, twodimensional echocardiography is mainly recommended to estimate left ventricular volume and ejection fraction (26). If three-dimensional echocardiography is available, it can avoid foreshortened views and result in better accuracy with regards to the assessment of left ventricular mass and volume (26). In addition, given that evaluations of valvular heart diseases such as aortic stenosis and mitral regurgitation are highly dependent on hemodynamic measurements, echocardiography with Doppler imaging is thus a useful tool (26). Further, novel techniques including twodimensional speckle tracking are currently available and can provide complementary information in the assessment of left ventricular function (26-28). The BACCARAT (BreAst Cancer and CArdiotoxicity Induced by RAdioTherapy) study, a 2-year follow-up prospective cohort of patients treated with breast radiotherapy, aims to study functional and anatomical cardiac imaging before, at the end of, and 6 and 24 months after radiotherapy (29). This ongoing trial will enhance the knowledge on the detection and prediction of early subclinical cardiac dysfunction and lesions induced by breast radiotherapy and on the potentially involved biological mechanisms (ClinicalTrials.gov: NCT0260512) (29).

Other imaging modalities, including cardiac computed tomography (CT), cardiac magnetic resonance (CMR), and nuclear cardiology, are used to confirm the diagnosis of RICVDs (26-28). Because cancer patients usually have to receive regular CT scans to evaluate the condition of their cancer, despite being less sensitive for coronary artery lesions than angiography using multi-detector computed tomography (MDCT), conventional or non-contrast CT may also help in the early detection of coronary or valvular calcification as well as pericardial diseases (26).

\section{Cardiovascular biomarkers before radiotherapy}

In addition to cardiovascular imaging, there is increasing focus on the use of biomarkers for the early detection of cardiotoxicity before it becomes irreversible (30-32). The most frequently used markers of cardiac injury are cardiac troponin and NT-proBNP (30-32). In 64 patients with breast cancer treated with a median dose of $60 \mathrm{~Gy}$, increased NT-proBNP concentrations were found from 9 months post radiotherapy to 24 months (33). Other inflammation markers including C-reactive protein, interleukin-6, galectin-3, and growth differentiation factor- 15 (GDF-15) are under investigation for their applicability in detecting RICVDs (30-32). In a prospective longitudinal study of 87 patients with breast cancer, lung cancer, or mediastinal lymphoma treated with photon or proton thoracic radiotherapy, although there were no significant increases in biomarker levels from pre-radiotherapy to postradiotherapy in the patients with breast cancer, elevated levels of placental growth factor (PIGF) and GDF-15 were found in those with lung cancer/lymphoma (34). Through screening 91 biomarkers in 342 breast cancer survivors, Tromp et al. found that several inflammatory biomarkers including GDF-15, monocyte chemoattractant protein 1, chemokine ligand 16, tumor necrosis factor super family member $13 \mathrm{~b}$ and proprotein convertase subtilisin/kexin type 9 were elevated in the survivors treated with chemoand radiotherapy, and that they were independently associated with lower left ventricular ejection fraction (35). Using microRNA arrays, genome-wide association studies and proteomics, novel markers of cardiovascular injury or inflammation have also been explored $(30,32,35,36)$.

\section{Screening and comprehensive follow-up evaluations}

A comprehensive long-term follow-up protocol for the early detection of RICVDs has yet to be established. Given that the epidemiological evidence does not provide clues on the key mechanisms underlying RICVDs, it is difficult to design an optimal preventive strategy. Currently, changing the radiotherapy field or targeted radiation with shielding of the heart remains one of the most important interventions to prevent RICVDs (37). Patients with classical cardiovascular risk factors should be treated aggressively $(1,12,19,22)$. Modifying risk factors including weight, smoking and hypertension may improve the long- 
term cardiovascular outcomes (12). Although the early detection and treatment of RICVDs can mitigate the associated mortality and morbidity, beyond cardiovascular screening at baseline, there is currently no practical protocol for follow-up evaluations post radiotherapy. Large and prospective studies designed with longitudinal followup for detecting cardiovascular sequelae before, during and after radiotherapy could facilitate to set up a risk-stratified score for precision management of cardiovascular risks among cancer patients.

\section{The strategy for patients who already have cardiovascular disease or received other anti- cancer therapies with cardiotoxicity}

Cancer patients with pre-existing myocardial dysfunction at baseline require a specialist cardio-oncology review, and preferably multi-discipline team care (38). Options include using alternative non-cardiotoxic chemotherapy, lower cardiotoxic liposomal doxorubicin, reduced-dose schedules, and precision location of the radiotherapy $(12,39)$. In addition, cardioprotective drugs such as angiotensin-converting-enzyme inhibitors or angiotensinreceptor blockers, beta-blockers, aldosterone antagonists or dexrazoxane may also be considered (24,40-42).

\section{Conclusions}

Advances in radiotherapy techniques have significantly decreased the incidence of RICVDs. However, cardiovascular complications are still more frequently observed in patients with left-sided breast cancer compared to those with right-sided breast cancer, suggesting that the risk remains. When treating a patient with thoracic radiotherapy, careful attention should be paid to risk factors that may contribute to subsequent cardiotoxicity. Cardiovascular evaluations and risk factor management can help to reduce subsequent cardiovascular complications. Even though no solid protocol for post-radiotherapy cardiovascular follow-up has yet been established, cardiovascular imaging to monitor coronary artery disease, cardiomyopathy, pericardial disease, valvular dysfunction, and conduction abnormalities is a reasonable approach. Cardiac biomarkers may also facilitate the early detection of myocardial dysfunction. However, large-scale randomized control studies are required to establish a clinical consensus.

\section{Acknowledgments}

We appreciate the support from the Taiwan Society of Cardiology and Chi-Mei Medical Center.

Funding: This work is supported by Chi-Mei Medical Center, Far Eastern Memorial Hospital, and the Ministry of Science and Technology of Taiwan (108-2314-B-418-002MY3, 110-2314-B-418-005-MY3).

\section{Footnote}

Provenance and Peer Review: The article was commissioned by the editorial office, Therapeutic Radiology and Oncology for the series "Cardio-Oncology". The article has undergone external peer review.

Reporting Checklist: The authors have completed the Narrative Review reporting checklist. Available at https:// dx.doi.org/10.21037/tro-21-21

Conflicts of Interest: All authors have completed the ICMJE uniform disclosure form (available at https://dx.doi. org/10.21037/tro-21-21). The series "Cardio-Oncology" was commissioned by the editorial office without any funding or sponsorship. YWW served as an unpaid Guest Editor for the series. The authors have no other conflicts of interest to declare.

Ethical Statement: The authors are accountable for all aspects of the work in ensuring that questions related to the accuracy or integrity of any part of the work are appropriately investigated and resolved.

Open Access Statement: This is an Open Access article distributed in accordance with the Creative Commons Attribution-NonCommercial-NoDerivs 4.0 International License (CC BY-NC-ND 4.0), which permits the noncommercial replication and distribution of the article with the strict proviso that no changes or edits are made and the original work is properly cited (including links to both the formal publication through the relevant DOI and the license). See: https://creativecommons.org/licenses/by-nc-nd/4.0/.

\section{References}

1. Cheng YJ, Nie XY, Ji CC, et al. Long-Term Cardiovascular Risk After Radiotherapy in Women With Breast Cancer. J 
Am Heart Assoc 2017;6:005633.

2. Clarke M, Collins R, Darby S, et al. Effects of radiotherapy and of differences in the extent of surgery for early breast cancer on local recurrence and 15-year survival: an overview of the randomised trials. Lancet 2005;366:2087-106.

3. Cuzick J, Stewart H, Rutqvist L, et al. Cause-specific mortality in long-term survivors of breast cancer who participated in trials of radiotherapy. J Clin Oncol 1994;12:447-53.

4. Darby SC, Ewertz M, McGale P, et al. Risk of ischemic heart disease in women after radiotherapy for breast cancer. N Engl J Med 2013;368:987-98.

5. Sylvester CB, Abe JI, Patel ZS, et al. Radiation-Induced Cardiovascular Disease: Mechanisms and Importance of Linear Energy Transfer. Front Cardiovasc Med 2018;5:5.

6. Cuomo JR, Sharma GK, Conger PD, et al. Novel concepts in radiation-induced cardiovascular disease. World J Cardiol 2016;8:504-19.

7. Raghunathan D, Khilji MI, Hassan SA, et al. RadiationInduced Cardiovascular Disease. Curr Atheroscler Rep 2017;19:22.

8. Kaidar-Person O, Zagar TM, Oldan JD, et al. Early cardiac perfusion defects after left-sided radiation therapy for breast cancer: is there a volume response? Breast Cancer Res Treat 2017;164:253-62.

9. Darby SC, McGale P, Taylor CW, et al. Long-term mortality from heart disease and lung cancer after radiotherapy for early breast cancer: prospective cohort study of about 300,000 women in US SEER cancer registries. Lancet Oncol 2005;6:557-65.

10. Yusuf SW, Venkatesulu BP, Mahadevan LS, et al. Radiation-Induced Cardiovascular Disease: A Clinical Perspective. Front Cardiovasc Med 2017;4:66.

11. Orzan F, Brusca A, Gaita F, et al. Associated cardiac lesions in patients with radiation-induced complete heart block. Int J Cardiol 1993;39:151-6.

12. Carver JR, Shapiro CL, Ng A, et al. American Society of Clinical Oncology clinical evidence review on the ongoing care of adult cancer survivors: cardiac and pulmonary late effects. J Clin Oncol 2007;25:3991-4008.

13. Yeh ET, Bickford CL. Cardiovascular complications of cancer therapy: incidence, pathogenesis, diagnosis, and management. J Am Coll Cardiol 2009;53:2231-47.

14. van Nimwegen FA, Schaapveld M, Janus CP, et al. Cardiovascular disease after Hodgkin lymphoma treatment: 40-year disease risk. JAMA Intern Med 2015;175:1007-17.

15. van Nimwegen FA, Ntentas G, Darby SC, et al. Risk of heart failure in survivors of Hodgkin lymphoma: effects of cardiac exposure to radiation and anthracyclines. Blood 2017;129:2257-65.

16. Fukada J, Shigematsu N, Takeuchi H, et al. Symptomatic pericardial effusion after chemoradiation therapy in esophageal cancer patients. Int J Radiat Oncol Biol Phys 2013;87:487-93.

17. Bradley JD, Paulus R, Komaki R, et al. Standard-dose versus high-dose conformal radiotherapy with concurrent and consolidation carboplatin plus paclitaxel with or without cetuximab for patients with stage IIIA or IIIB non-small-cell lung cancer (RTOG 0617): a randomised, two-by-two factorial phase 3 study. Lancet Oncol 2015;16:187-99.

18. Larsen RL, Jakacki RI, Vetter VL, et al. Electrocardiographic changes and arrhythmias after cancer therapy in children and young adults. Am J Cardiol 1992;70:73-7.

19. Doyle JJ, Neugut AI, Jacobson JS, et al. Radiation therapy, cardiac risk factors, and cardiac toxicity in earlystage breast cancer patients. Int J Radiat Oncol Biol Phys 2007;68:82-93.

20. Hooning MJ, Botma A, Aleman BM, et al. Long-term risk of cardiovascular disease in 10-year survivors of breast cancer. J Natl Cancer Inst 2007;99:365-75.

21. Hardy D, Liu CC, Cormier JN, et al. Cardiac toxicity in association with chemotherapy and radiation therapy in a large cohort of older patients with non-small-cell lung cancer. Ann Oncol 2010;21:1825-33.

22. Giordano SH, Kuo YF, Freeman JL, et al. Risk of cardiac death after adjuvant radiotherapy for breast cancer. J Natl Cancer Inst 2005;97:419-24.

23. Marinko T. Pericardial disease after breast cancer radiotherapy. Radiol Oncol 2018;53:1-5.

24. Groarke JD, Nguyen PL, Nohria A, et al. Cardiovascular complications of radiation therapy for thoracic malignancies: the role for non-invasive imaging for detection of cardiovascular disease. Eur Heart J 2014;35:612-23.

25. McGale P, Darby SC, Hall P, et al. Incidence of heart disease in 35,000 women treated with radiotherapy for breast cancer in Denmark and Sweden. Radiother Oncol 2011;100:167-75. 
26. Lancellotti P, Nkomo VT, Badano LP, et al. Expert consensus for multi-modality imaging evaluation of cardiovascular complications of radiotherapy in adults: a report from the European Association of Cardiovascular Imaging and the American Society of Echocardiography. J Am Soc Echocardiogr 2013;26:1013-32.

27. Tuohinen SS, Skytta T, Huhtala H, et al. Left Ventricular Speckle Tracking Echocardiography Changes Among Early-stage Breast Cancer Patients Three Years After Radiotherapy. Anticancer Res 2019;39:4227-36.

28. Walker V, Lairez O, Fondard O, et al. Early detection of subclinical left ventricular dysfunction after breast cancer radiation therapy using speckle-tracking echocardiography: association between cardiac exposure and longitudinal strain reduction (BACCARAT study). Radiat Oncol 2019; 14:204.

29. Jacob S, Pathak A, Franck D, et al. Early detection and prediction of cardiotoxicity after radiation therapy for breast cancer: the BACCARAT prospective cohort study. Radiat Oncol 2016;11:54.

30. Ananthan K, Lyon AR. The Role of Biomarkers in CardioOncology. J Cardiovasc Transl Res 2020;13:431-50.

31. Pudil R, Mueller C, Čelutkienè J, et al. Role of serum biomarkers in cancer patients receiving cardiotoxic cancer therapies: a position statement from the Cardio-Oncology Study Group of the Heart Failure Association and the Cardio-Oncology Council of the European Society of Cardiology. Eur J Heart Fail 2020;22:1966-83.

32. Riddell E, Lenihan D. The role of cardiac biomarkers in cardio-oncology. Curr Probl Cancer 2018;42:375-85.

33. D'Errico MP, Grimaldi L, Petruzzelli MF, et al. N-terminal pro-B-type natriuretic peptide plasma levels as a potential biomarker for cardiac damage after radiotherapy in patients with left-sided breast cancer. Int J Radiat Oncol Biol Phys 2012;82:e239-46.

34. Demissei BG, Freedman G, Feigenberg SJ, et al. Early

doi: $10.21037 /$ tro-21-21

Cite this article as: Chang WT, Feng YH, Chen ZC, Wu YW. Narrative review-cardiovascular evaluation before radiotherapy for patients with breast cancer and other malignancies. Ther Radiol Oncol 2021;5:24.
Changes in Cardiovascular Biomarkers with Contemporary Thoracic Radiation Therapy for Breast Cancer, Lung Cancer, and Lymphoma. Int J Radiat Oncol Biol Phys 2019;103:851-60.

35. Tromp J, Boerman LM, Sama IE, et al. Long-term survivors of early breast cancer treated with chemotherapy are characterized by a pro-inflammatory biomarker profile compared to matched controls. Eur J Heart Fail 2020;22:1239-46.

36. Hamam R, Hamam D, Alsaleh KA, et al. Circulating microRNAs in breast cancer: novel diagnostic and prognostic biomarkers. Cell Death Dis 2017;8:e3045.

37. Yusuf SW, Sami S, Daher IN. Radiation-induced heart disease: a clinical update. Cardiol Res Pract 2011;2011:317659.

38. Chang WT, Feng YH, Kuo YH, et al. The impact of a multidisciplinary cardio-oncology programme on cardiovascular outcomes in Taiwan. ESC Heart Fail 2020;7:2135-9.

39. Chiang CE, Wang TD, Lin TH, et al. The 2017 Focused Update of the Guidelines of the Taiwan Society of Cardiology (TSOC) and the Taiwan Hypertension Society (THS) for the Management of Hypertension. Acta Cardiol Sin 2017;33:213-25.

40. Brown SA, Okwuosa TM, Barac A, et al. The Role of Angiotensin-Converting Enzyme Inhibitors and $\beta$-Blockers in Primary Prevention of Cardiac Dysfunction in Breast Cancer Patients. J Am Heart Assoc 2020;9:e015327.

41. De Giorgi U, Giannini M, Frassineti L, et al. Feasibility of radiotherapy after high-dose dense chemotherapy with epirubicin, preceded by dexrazoxane, and paclitaxel for patients with high-risk Stage II-III breast cancer. Int J Radiat Oncol Biol Phys 2006;65:1165-9.

42. Hellmann K. Dexrazoxane and the ASCO guidelines for the use of chemotherapy and radiotherapy protectants: a critique. J Clin Oncol 2000;18:2004-6. 